\title{
The characterisation of the onset of plasticity through thin film inflation
}

\author{
Khurram Suleman* and Federico Bosi \\ Department of Mechanical Engineering, University College London, London, UK \\ *Email: khurram.suleman.18@ucl.ac.uk
}

\begin{abstract}
Structural membranes are ubiquitous due to their ultralow weight and ability to undergo large deformations, with applications ranging from novel civil constructions to advanced aerospace systems, and including biomedical devices, soft robotics, and tissue engineering. Although they are widely employed to withstand severe hygrothermal and mechanical loading conditions [1], the remarkably incomplete understanding of their governing deformation mechanisms before, after, and at the onset of yielding represents a limit in the development of such innovative systems [2].

In this work, we present a new experimental method, based on the inflation of plane membranes, to characterise the yield strength of elastomeric thin films, thus overcoming the limitations of the available empirical techniques. The phenomenon of strain localisation during inflation, which has not been utilised before, is employed to identify the material yield domain. The framework is developed by obtaining a finite strain analytical solution for the inflation of elastoplastic thin films that is numerically validated by means of finite element simulations.
\end{abstract}

The proposed technique is graphically illustrated in Figure 1 with the help of strain contours obtained by the analytical method and FEA. A hypothetical bilinear elastoplastic material, Figure 1(a), with elastic modulus $E$, yield stress $\sigma_{y}$ and a tangent modulus $E_{t}$ in the plastic phase of the material is used as an example. The accumulation of strain at the centre is evident in Figure 1(c) and (d) after the occurrence of plasticity, which was maximum on the edges during the elastic phase, Figure 1(b). Through the digital image correlation technique, experiments are performed for several materials on a custom-made setup and they prove the accuracy and reliability of the proposed technique in assessing the onset of the plasticity of highly deformable membranes. Therefore, such observed localisation can be employed for the development of a novel imaging technique to assess the yield strength in thin films.

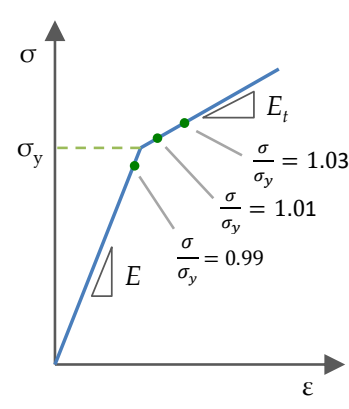

a)

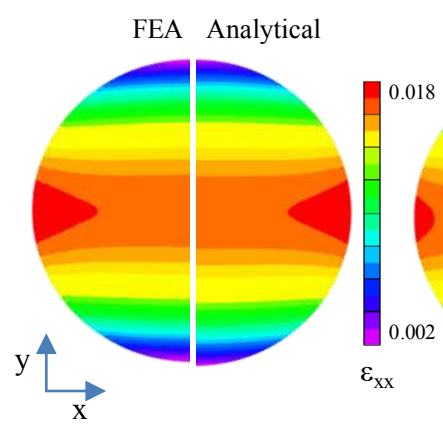

b)

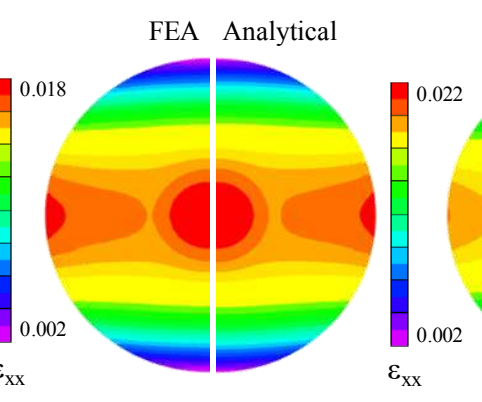

c)

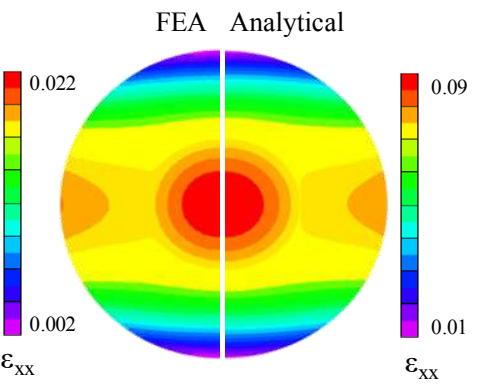

d)

Figure 1: a) Uniaxial stress-strain curve for a bilinear elastoplastic material, contour plots of horizontal strain $\varepsilon_{x x}$ from FEA and analytical results for b) $\sigma / \sigma_{y}=0.99$, c) $\sigma / \sigma_{y}=1.01$ and d) $\sigma / \sigma_{y}=1.03$, showing strain localisation at the centre of membrane at the onset of plasticity.

\section{REFERENCES}

[1] F. Bosi, S. Pellegrino. Nonlinear thermomechanical response and constitutive modelling of viscoelastic polyethylene membranes. Mechanics of Materials, 117, 9-21, 2018.

[2] F. Bosi, S. Pellegrino. Molecular based temperature and strain rate dependent yield criterion for anisotropic elastomeric thin films, Polymer 125, 144-153, 2017. 